\title{
Une certaine idée de la recherche en
} communication

\section{Hugues Hotier}

\section{(2) OpenEdition}

1 Journals

Édition électronique

URL : http://journals.openedition.org/communicationorganisation/2978

DOI : 10.4000/communicationorganisation.2978

ISSN : $1775-3546$

\section{Éditeur}

Presses universitaires de Bordeaux

\section{Édition imprimée}

Date de publication : 1 mai 1994

ISSN : 1168-5549

\section{Référence électronique}

Hugues Hotier, "Une certaine idée de la recherche en communication », Communication et organisation [En ligne], HS N${ }^{\circ} 1$ | 1994, mis en ligne le 27 mars 2012, consulté le 21 septembre 2020. URL : http:// journals.openedition.org/communicationorganisation/2978; DOI : https://doi.org/10.4000/ communicationorganisation. 2978

Ce document a été généré automatiquement le 21 septembre 2020.

(c) Presses universitaires de Bordeaux 


\title{
Une certaine idée de la recherche en communication
}

\author{
Hugues Hotier
}

1 L'institut des Sciences de l'Information et de la communication (ISIC) est une unité de formation et de recherche de l'université Michel de Montaigne-Bordeaux 3. Avec un effectif réduit, environ 250 étudiants, et un corps enseignant disponible et actif, l'ISIC est une véritable école universitaire. Nous avons coutume de parler à son propos d'esprit école et de culture universitaire. C'est là, presque, notre slogan. En tout cas, ça l'est sans doute au sens étymologique du terme : le cri de ralliement de la tribu. Ayant opté, dès sa fondation par Robert Escarpit il y a un quart de siècle, pour la professionnalisation fondé sur un haut niveau conceptuel, l'ISIC comprend un institut universitaire professionnalisé (IUP), qui délivre la maîtrise et le titre d'ingénieurmaître, et un diplôme d'études supérieures spécialisées (DESS) de troisième cycle.

2 L'ISIC est une structure universitaire et, par conséquent, la recherche y tient une place prépondérante. Fondé en 1986, le Groupe de Recherche en Communication des Organisations (GREC/O) rassemble seize chercheurs et des doctorants en nombre variable qui travaillent à la fois sur des problématiques théoriques et sur des sujets de recherche appliquée. Ces derniers étant souvent proposés par des entreprises ou par d'autres organisations avec lesquelles des contrats et des conventions sont signés. Il est remarquable que sur les seize chercheurs du GREC/O deux soient des professionnels, non universitaires, titulaires d'un doctorat -l'une est parisienne et exerce une activité de consultante, l'autre, aquitain, est ingénieur dans un centre d'essai aéronautique. Quatre des quatorze chercheurs universitaires du GREC/O appartiennent à des universités géographiquement éloignées de Bordeaux. Ils viennent de Lille, Le Mans, Paris et Reims. Les Bordelais ne sont pas peu fiers d'avoir été ainsi rejoints par des collègues attirés par leurs travaux.

3 Nous pensons que le chercheur a définitivement cessé d'être un solitaire retiré dans une tour d'ivoire où il pense supérieurement et superbement. Cette vision romantique des choses, si tant est qu'elle ait jamais eu un fondement réaliste, a fait long feu. D'abord les chercheurs travaillent en équipe et, c'est notre cas, en équipes 
pluridisciplinaires le plus souvent. De plus, ils sortent fréquemment des limites de leur groupe pour aller consulter d'autres chercheurs qui ont des compétences dans des domaines autres que les leurs. Ensuite, la recherche telle que nous l'entendons procède d'une conception moderne de l'université : il est fini le temps où les professeurs se drapaient dans la dignité de leur toge pour n'avoir de comptes à rendre à personne. L'université doit participer au développement du pays qui lui permet d'exister. Elle doit non seulement faire progresser la connaissance mais elle doit aussi participer à l'exploitation des savoirs nouveaux qu'elle a contribué à mettre au monde. En ce sens, l'Etat est en droit de demander à l'université de contribuer au développement du pays et de la nation.

Qu'on nous comprenne bien: le devoir que nous définissons est d'essence morale et l'université ne doit pas cesser d'être un lieu de liberté car le progrès intellectuel ne saurait s'accommoder de la contrainte imposée par des politiques trop souvent à courte vue et vite périmées. Disons pour faire vite que nous prônons une université utile plutôt qu'utilitaire, productive plutôt que productiviste.

C'est dans cet esprit que le Groupe de Recherche en Communication des Organisations travaille et produit. Et, loin de la tour d'ivoire évoquée ci-dessus, les chercheurs qui le composent estiment qu'ils ont aussi à remplir une fonction de dynamisation et d'animation. Ils pensent qu'il est de leur devoir de susciter la réflexion sur le champ disciplinaire qui est le leur. C'est pourquoi, ils publient depuis 1992 une revue scientifique, Communication \& Organisation, qui est plus un lieu de confrontation des idées et d'innovation intellectuelle que d'étalage de connaissances. C'est pourquoi aussi, depuis 1987, ils organisent des séminaires ouverts et des colloques qui réunissent systématiquement praticiens et chercheurs. De la communication interne des entreprises à la communication interculturelle, de la relation tumultueuse entre l'éthique et la communication à la communication institutionnelle des grandes organisations, nos colloques analysent tous les domaines que la communication a investis. Tous les domaines et tous les lieux. Tous les lieux et tous les mondes.

Et, aujourd'hui, c'est à la fois d'un lieu et d'un monde qu'il est question car hôpital est un mot qui désigne en même temps ce bâtiment qui inquiète et réconforte, et au large duquel on espère toujours pouvoir passer, et une société où voisinent des populations fort différentes les unes des autres : les malades, les médicaux, les administratifs. Des groupes humains qu'on imagine homogènes et fermés et que les stéréotypes dépeignent comme exigeants, généreux ou contraignants. On comprend combien ce monde a besoin de la communication et combien aussi celle-ci doit inquiéter.

7 Un colloque comme celui-ci est des plus importants et nous souhaitons qu'il aide à démystifier autant qu'à démythifier. Nous souhaitons qu'il aide à comprendre et à se comprendre. Et qu'il fasse progresser la connaissance pour que s'instaure ou se maintienne une harmonie indispensable dans un lieu où des gens souffrent et meurent.

Je ne voudrais pas que cette introduction se transforme en panégyrique. Mais je ne voudrais pas non plus faire preuve d'ingratitude ni, d'ailleurs, bouder le plaisir que j'aurai à remercier et à féliciter les personnes qui ont préparé ce colloque. Je le ferai brièvement mais sincèrement et, pardonnez ce mot peut-être incongru, joyeusement. Avec la joie de celui qui constate qu'il vit dans un monde positif au milieu de gens exceptionnels. Je voudrais donc très simplement remercier Valérie Carayol, maître de conférences à l'ISIC, qui a assumé la direction scientifique et la coordination ce 
colloque, ainsi que nos collègues Catherine Pascal et Christophe Bultel qui l'ont efficacement secondée.

9 Et le directeur que je suis s'en voudrait de ne pas mentionner que la conception de la logistique, l'organisation matérielle et la communication de ce colloque sont l'œuvre des étudiants du DESS en communication des organisations qui administrent ainsi la preuve que l'on peut tout à la fois, être apte à produire des idées et à leur donner forme.

\section{AUTEUR}

\section{HUGUES HOTIER}

Professeur des universités Directeur de l'ISIC et du CREC/O 